\title{
A survey of Video Watermarking based on Motion Vector
}

\author{
Liu Yan', Zhou Ping ${ }^{2}$ \\ Guilin University of Electronic Technology, Guangxi, China \\ ${ }^{1}$ liuyan89510501@163.com, ${ }^{2}$ jingzhou@ guet.edu.cn
}

\begin{abstract}
Digital watermarking is the process of embedding information into a digital signal in a way that is difficult to remove. The digital watermarking for video is an effective method to protect the video copyright. This paper introduces the basic classification of video watermarking and the basic concepts of the motion vector, and introduces a combination of motion vectors with different compression standards to produce a variety of video watermarking algorithms and analyze its advantages and disadvantages. Analyze some kinds of attacks of the video watermarking, summarize and looking out the future of video watermarking based on motion vector.

Index Terms - video watermarking, motion vectors, watermarking algorithm, the attacks
\end{abstract}

\section{Introduction}

The digital video watermarking technology is that the information which embedded in the video works can mark copyright of the authors. Because the watermark information is to use the redundant information of the image as a carrier, the watermark is eliminated as the redundant information in the process of image compression; therefore, a watermark is embedded in the specific information in the video image. Compared with still images, the difference of the moving images lie in the movement of the video sequence, in the video coding, this movement is performance in the motion vector. Thus embed watermark information in motion vector become a research direction.

\section{Life Cycle and Classification of Video Watermarking}

A complete digital watermarking system should include three basic parts [1]: watermark generation, watermark embedding and watermark extraction. Embedding and extraction diagram of digital video watermarking is as follows:

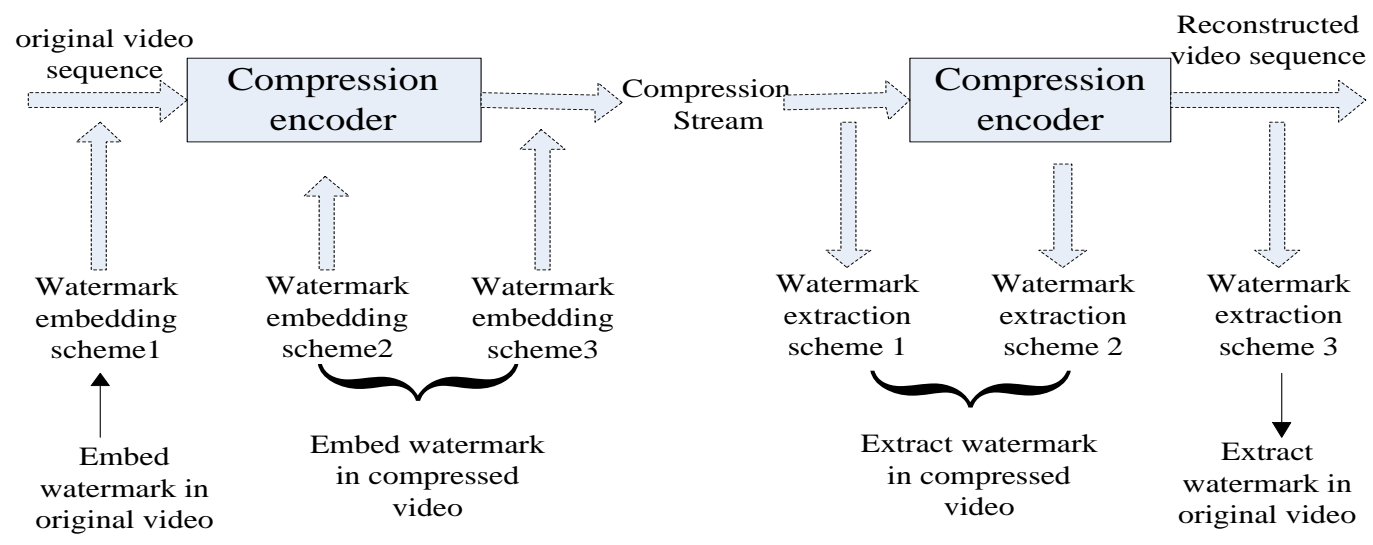

Figure 1. Digital video watermarking embedding and extraction scheme.

According to the different ways of the combination of digital video watermark technology and coding system to classify, the classification ${ }^{[2]}$ include the method based on the original video and the method based on compressed video.

Reference [2] mentioned that the watermarking algorithm based on the original video processed the video data without encode, Embedded watermark in the original video data, called the pre-video watermarking algorithm. The watermarking algorithm based on the compressed video combined with some kind of video compression standards, embed watermark in the compressed video, called built-in video watermark algorithm and rear video watermark algorithm. The algorithm that embed watermark in the video coding structure is the built-in video watermarking algorithm, other algorithm that embed watermark in the bit stream by video encoding is the rear video watermarking algorithm. The motion vector is carried in the compressed stream, therefore, by modifying the motion vectors to embed the watermark belongs to the rear video watermarking algorithm.

\section{Basic Concept of Motion Vectors}

In the inter-frame predictive coding, the scene in the adjacent frames of the moving images has a certain correlation, so divide the moving images into blocks or macroblocks, and try to search out the location of each block or macroblock in the neighboring frames, get the relative offset of the spatial location between the two. The relative offset is commonly referred to as motion vectors, the process to get motion vectors called motion estimation [3]. 
Motion vector and the prediction error which get from the motion match are sent to the decoder, in the decoder according to the position specified by the motion vector, find out the corresponding block or macroblock from the adjacent reference frame image has been decoded, sum up the position and the prediction error to get the position of the block or macroblock in the current frame.

Motion vector reflect the information of motion displacement of the pixel marcoblock. it relates to the time continuity and smoothness of the video screen, and it is an important object for the video compression. In the video encoding data losses small, it is suitable for hidden watermark[4].

Motion estimation can remove the inter-frame redundancy, and reduce the number of bits of the video transmission. Therefore, motion estimation is an important part in the video compression and procession system.
Digital Video Watermarking Algorithm based on a combination of motion vectors with different compression algorithms

Video watermarking embeds the watermarking information into the redundancy data of the video data, to protect the copyright or completeness of the digital products. As the data of the original video is very large, generally speaking video watermark is embedded in the compressed domain. Different coding standards use different compression method, so combination of a specific coding standard to embed video watermark.

Watermarking algorithm based on motion vectors in MPEG Compressed Domain. Such algorithms embed the watermark information in the MPEG compressed stream directly without going through the process of encoding and decoding, almost no effect on the quality of the video image. But the bit rate of the compressed video sequence constrains the capacity of the watermark, because there is no enough data redundancy in the compressed bit stream. The flow chat of the processing algorithm is as follows:

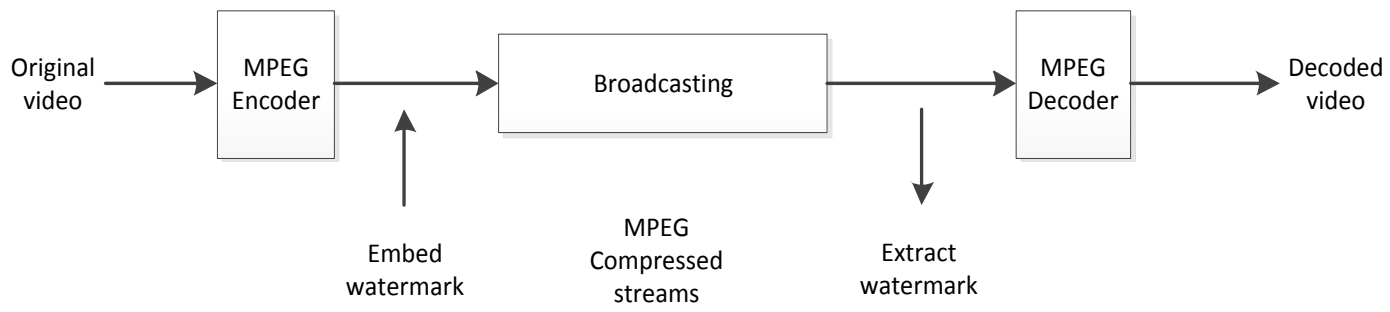

Figure 2. The flow chat of the processing algorithm of MPEG video watermark.

Jordan[5]proposed a video watermarking algorithm based on motion vector at first, the algorithm to embedded watermark information by modifying the motion vectors of MPEG-4 video steam, according to the key all the motion vectors looked as the embedded point, although this reduces the computational complexity of watermark embedding, reduces the robustness and imperceptibility of the watermark.

Zhang[6]made some improvement to the Jordan's algorithm, according to the principle of the human visual system has a lower sensitivity to the faster velocity region, set a threshold for the motion vector, select the value of the amplitude greater than the threshold of motion vector to embed watermark information. And different watermark information embedded in the different pieces of motion vector, the algorithm enhances the robustness of the watermark.

Dai[7]proposed watermark algorithm based on the Game of Life scrambling MPEG-4 motion vector, used Game of Life scrambling method to preprocess the watermark information, and considered the motion vector amplitude and texture features of the video image to embed watermarking adaptively, make the watermark algorithm and the video coding fully combine, improve the ability to resist the compressed.
Watermarking algorithm based on motion vectors for H.264. ITU-T adopted H.264 as international standard formally in 2003[8], the promulgation of the H.264 is a major event in the development of video compression coding, its superior compression performance will also play an important role in various aspects, such as digital television broadcasting, video, real-time communication, network video streaming delivery and multimedia messaging etc. Therefore, many scholars have carried out deep research.

Zheng zhendong[9] proposed a video watermarking scheme for H.264 which is a new generation of video compression standard. Use motion vector regional characteristics to hide the watermark information. In the algorithm using the motion estimation search algorithm in the H.264 encoding process, to embed watermark information according to the motion vector relative to the current macroblock position of regional. The algorithm has low computational complexity and good ability to resist geometric attack.

Najla Mohaghegh[10] proposed a video watermarking scheme which embeds copyright information in $\mathrm{H} .264$ bit stream motion vectors. In the proposed method, a watermark string is embedded into the motion vector components with the 
larger size considering the video movement direction which will be retrieved in decoder. The main contribution is to adjust an adaptive threshold in such a way that with a defined number of embedded bits, i.e. the watermark payload, the video quality degradation will be negligible.

Ma fuzhu[11]proposed a video watermarking algorithm based on the difference of component the motion vector. Use the special tree structure motion compensation mechanism in H.264 and a quarter pixel motion estimation, combine with the idea of differential energy watermarking algorithm, at the same time take full account of the human visual characteristics, embed watermark by controlling the difference between the horizontal and vertical components of each of motion vector. This algorithm embedded watermark by selectively change the motion vector horizontal component values, to ensure that the watermark embedding capacity, to ensure the visual quality of the video, and watermark detection did not require the original video. The experimental result showed that, the algorithm was simple, fast, effective, able to meet the requirements of realtime processing of video, can resist the H.264 compression attacks, to achieve the purpose of the video copyright protection.

The main features of video watermarking scheme based on motion vectors are as follows:

(1) Basically, the watermark information is embedded directly in the stream, this will not increase the coding complexity.

(2) Watermark information extraction is very simple, just do a simple calculation of the coordination values of the motion vector.

(3) Embedded watermark dose not influence the quality of the video image.

(4) Watermark capacity is adjustable.

The watermarking algorithm combined with different compression algorithms has a fatal shortcoming, it is that if compression algorithm changes, there may not be able to extract the watermark.

\section{The Attack of Video Watermark}

It is necessary to take full account that the watermarking system could be influenced by various kinds of attacks when it is applied in the copyright protection, content the authentication and so on. Of course, the watermark abilities against the attack in different application areas have different requirements, and the ability against the attack is the most important performance of the Digital watermarking evaluation system. Compared to the embedding algorithm of watermark, the research of the watermark attack hasn't formed a system and there is no a watermarking scheme can resist any attacks.

The attack of video watermark is a video process which could weaken the watermark detection or cause polysemy to the watermark message. Deguillaume and Hartung ${ }^{[12,13]}$ divide attacks of video watermark into simple attack, desynchronized attack, confused attack and removing watermark attack. The purpose of attacks is to make the corresponding testing tools of the video watermark system can not recover watermarking signal or detect watermarking signal existence. The following are some common attacks of video watermark which are introduced from different sides and ways.

(1) Simple attack The simple attack generally refers to unintentional attack, which is some conventional video processing methods' make influence on the watermark information. Such as the loss of watermark information when coding video with all kinds of compression coding standards; the changes of the frame rate and spatial resolution when NTSC PAL and SECAM convert with video standard format; the change of the screen display formats such as 4/3, 16/9 and 2.11/1; the video editing processing of frame deletion, frame insertion and frame reintegration, the geometric distortion of video brought by digital-to-analogue conversion and analogueto- digital conversion, all of this will make the video watermark be destructed.

(2)Desynchronized attack For most of the watermarking technologies, it is necessary to know the location of embedded watermark. Desynchronized attack is to destroy the synchronicities of the video sequences and watermark on space or time, in order to make the detection algorithm cannot detect watermarking information. The desynchronized attack generally adopts geometry transform, such as zoom, the translation of space direction, the translation of time direction (video digital works), rotation, shear, pixel displacement, second sampling, insertion or extraction of pixels or cluster of pixels, etc.

(3) Confused attack The confused attack produces confusion by means of mendacious watermark information or original video sequence, which is put forward by Craver $^{[14]}$ in IBM and known as IBM attack. The principle is that the attackers defines faked watermark as reference model which randomly generated, and then subtract the reference model from the works with watermark, finally a faked work is generated. Or embeds one or more additional watermark into the image already having watermark in order to confuse the first watermark containing copyright information, then the copyright becomes uniqueness. By this way, the attacker could state copyright of the original works and the copyright protection function of digital watermark is challenged.

(4)Removing watermark attack By ways of analyzing the watermark data and estimating the watermark information in video image, the removing watermark attack separates the video image with the watermark information and abandons watermarking information, then gets video image without containing watermark information, finally achieves the purpose of illegal theft. The common removing attack methods are collusion attacks, denoising, determinate nonlinear filtering, compression with image comprehensive model, and so on.

How to design a preferable video watermark project to resist a variety of deliberate and indeliberate attacks is the important and difficult point of video watermark technology. To design a robust video watermark algorithm according to these attack methods becomes the development direction of video watermark technology. 


\section{Conclusion}

Video storage and transmission are in the compressed stream, with the development of compression algorithms, embedding watermark in the compressed video stream is the hotspot in scholars' research. Motion vector is important information carried by the compressed stream of video. The motion vector is one of the most important technologies in motion estimation and motion compensation of video, through motion estimation algorithm of video coding to obtain. motion vector reflects the motion displacement information between the predicted macroblock in the current encoded frame and the best match macroblock in the reference frame, noting to do with special content of the macroblock, so it has strong robustness with the video watermark attacks based on the content. After analyze several watermark attack, we should focus on the digital video watermarking algorithm based on motion vector in different compression algorithm, improve its ability to resist specific attacks.

\section{Acknowledgements}

This work was financially supported by the National Natural Science Foundation of China (60961002) and the Natural Science Foundation of Guangxi (2012GXNSFAA053221).

\section{References}

[1] Kong X W, Liu Y, Liu H J and Yang D L. 2004 Object watermarks for digital images and video. Image and Vision Computing; 22(08):583595.

[2] Sun SH H, Lu ZH M and Niu X M. 2004 Digital watermarking technology and application Beijing: Science press
[3] Bi H J. 2005 A new generation of video compression coding standardh.264 / AVC. Beijing: PSOTS\&TELECOM PRESS

[4] Chen X. 2011_Application and Research on Moving Object Detection Technology in Video Watermarking. Guangxi: Guilin university of electronic technology

[5] F.Jordon, M.Kutter, T.Ebrahimi. 1997 Proposal of watermarking technique for hiding/ retrieving data in compressed and decompressed video. ISO/IEC, JTCI/SC29/WG11, 241-246

[6] Zhang J, Li J G, Zhang L. 2001 Video watermark technique in motion vector. XIV Brazilian Symposium on Computer Graphics and Image Processing, 179-182

[7] Dai K F, Huang W Y. 2004 watermark algorithm based on the Game of Life scrambling MPEG-4 motion vector. ACTA SCIENTIARUM NATURALIUM UNIVERSITATIS SUNYATSENI, 43(11):192-195

[8] Iain E.G.Richardson. 2004 H.264 and mpeg-4 video compression. Changsha: National University of Defense Technology Press

[9] Zheng Zh D, Wang P, Chen S. 2008 A Video Watermarking Scheme Based on the Region

[10] Character of Motion Vectors. Journal of Image and Graphics, 13(10):1926-29

[11] Najla Mohaghegh. 2008 H.264 Copyright Protection with Motion Vector Watermarking IEEE 1384-89

[12] Ma F Zh, Chen D. 2009 Differential Component Video Watermarking Algorithm Based on Motion Vector. Guang Dong: China Institute of Communications.134-138

[13] F.Hartung, J.K.Su, B.Girod. 1999 Spread spectrum watermarking: malicious attacks and counterattacks. Security and watermarking of Multimedia Contents, Proceeding of SPIE, 36(57):147-158

[14] F.Deguillaume, G.Csurka, T.Pun. 2000 Countermeasures for unintentional and intentional video watermarking attacks. Security and Watermarking of Multimedia Contents II, Proceeding of the SPIE, 39:346-357

[15] S.Craver, N.Memon, B.L.Yeo, M.M.Yeung. 1998 Resolving rightful ownerships with invisible watermarking techniques: Limitations, Attacks and Implications. IEEE Journal on Selected Areas in Communications, 16(4): 573-586 